\title{
Retos y nivel de satisfacción de los estudiantes del subsistema de universidades tecnológicas ante la nueva modalidad de educación en línea
}

\author{
Herrera Cárdenas Jorge Antonio \\ jorgeahc@live.com.mx \\ Departamento de Investigación y Desarrollo
}

Merino Viazcan Janet

jafe174@hotmail.com

Programa Educativo de Administración

Bravo Aguirre Ariadna Guadalupe

ariadna.bravo@utim.edu.mx

Programa Educativo Procesos Alimentarios

Castillo Sanguino Narciso

narciso.castillo@utim.edu.mx

Programa Educativo de Lengua Inglesa

Universidad Tecnológica de Izúcar de Matamoros, Prolongación Reforma 168 Barrio de Santiago Mihuacán, Izúcar de Matamoros Puebla C.P. 74420

\section{RESUMEN}

Ante la actual contingencia derivada por la pandemia de Covid-19, la educación en línea fue la única alternativa para no retrasar los programas educativos. Aunque las limitaciones son comunes en la mayoría de las instituciones educativas, son más acentuadas en las comunidades rurales. El objetivo de este trabajo fue identificar y comparar los retos y dificultades entre los estudiantes del SUTyT de las comunidades rurales vs instituciones ubicadas en regiones más urbanizadas en su formación con modelo de aprendizaje virtual. Se estructuró un instrumento de encuesta de 32 ítems, que se aplicó a estudiantes de tres instituciones del SUTyT: UTIM; Izúcar de Matamoros (rural), UTP; Puebla y UTJ; Jalisco (urbanas). Las respuestas se organizaron y analizaron estadísticamente para identificar los principales retos y problemáticas, así como las causas. Los resultados 
mostraron que los estudiantes de la UTIM perciben menores ingresos económicos y aportan a la economía familiar; los estudiantes de las tres instituciones coincidieron en la falta de un dispositivo electrónico adecuado y de conexión de internet en sus hogares, por la falta de cobertura o de ingresos insuficientes para pagar el servicio. Se concluye que la brecha tecnológica derivada de las carencias económicas pone en desventaja a los estudiantes ante este modelo de enseñanza, acentuándose en aquellas comunidades alejadas de la mancha urbana.

Palabras clave: educación virtual; covid-19; retos de aprendizaje virtual. 


\title{
Challenges and satisfaction level of the students of the technological universities subsystem to the new modality of online eduation
}

\begin{abstract}
Due to the current contingency resulting from COVID-19 pandemic, online education was the only alternative to avoid delaying educational programs. Although limitations are common in most educational institutions, they are more accentuated in rural communities. This paper identified and compared the challenges and difficulties among Technological Universities Subsystem's students from rural communities vs. institutions located in more urbanized regions in their training with a virtual learning model. A 32-item survey instrument was structured and applied to students from three institutions: UTIM; Izúcar de Matamoros (rural), UTP; Puebla and UTJ; Jalisco (urban). The responses were organized and statistically analyzed to identify the main challenges, problems, and causes. The results showed that UTIM students perceive lower economic income and contribute to the family economy; students from the three institutions agreed in the lack of an adequate electronic device and Internet connection at home, due to the lack of WIFI coverage or insufficient income to afford the service. It is concluded that the technological gap derived from economic deprivation puts students at a disadvantage when faced with this teaching model, accentuated in those communities far from the urban area.
\end{abstract}

Keywords: online education; covid-19; online learning challenges.

Artículo recibido: 30 noviembre. 2021 Aceptado para publicación: 29 diciembre 2021

Correspondencia: jorgeahc@live.com.mx Conflictos de Interés: Ninguna que declarar 


\section{INTRODUCCIÓN}

El Subsistema de Universidades Tecnológicas (SUTyP) se estableció hace 30 años con la finalidad de una mayor equidad e igualdad social y de índole económica, que relacionan la educación con el crecimiento económico y con una estrategia de competitividad de los sectores productivos. Además de introducir un subsistema público a nivel superior que permitiera que en dos años el estudiante obtuviera un título de técnico superior universitario (CGUT, 2000 en Arenas, 2009).

El Covid-19 es una enfermedad causada por una nueva especie de coronavirus que fue descubierta en la ciudad de Wuhan China a finales del 2019 (Guach et al., 2020). En México se detectó el primer caso de Covid-19 el 27 de febrero de 2020 (Suarez et al., 2020). Ante la gravedad de la pandemia, el 2 de marzo de 2020 se publicó en el Diario Oficial de la Federación un acuerdo donde se informó la suspensión de clases presenciales en todos los niveles educativos, como una de las estrategias para evitar la propagación de la enfermedad (Fernández et al., 2020).

En el sector educativo a nivel mundial, más de 1,200 millones de estudiantes dejaron de asistir a clases presenciales, de ellos 160 millones en América Latina (Cepal/Unesco 2020). Tan solo en la Universidad Tecnológica de Izúcar de Matamoros (UTIM), fueron 1729 estudiantes los que siguieron sus clases en línea. La suspensión obligatoria de las actividades educativas ha generado múltiples efectos en todos los actores del proceso educativo. A nivel superior se han tenido que tomar diversas medidas para acatar las indicaciones del gobierno federal y estatal. Al tiempo que se intenta facilitar a los profesores la infraestructura y las herramientas tecnológicas para continuar con las actividades académicas a distancia (Mendiola et al., 2020). Bajo esta nueva normalidad, los países en desarrollo enfrentan un gran reto para el manejo de un escenario cambiante y repentino de la planificación, gestión y organización educativa durante esta pandemia con su infraestructura técnica fracturada, incompetencia académica y falta de recursos (Thomas, 2020 en Mishra et al., 2020). Actualmente, menos de la mitad de la población en Latinoamérica y el Caribe tiene conexión a banda ancha fija y solo el 9,9 \% cuenta con fibra de alta calidad en el hogar. El $87 \%$ de la población vive dentro del alcance de una señal de 4G, el uso y la penetración reales siguen siendo bajos (37 \%). Solo el 40\% de la población rural en Latinoamérica tienen opciones de conectividad en comparación con el $71 \%$ de la población de zonas urbanas (Dress y Zhang, 2021). 
La brecha tecnológica acentúa los factores de conectividad, dispositivos, apropiación de tecnología e inadecuada formación docente para el uso de tecnologías de la información (Figueroa et al., 2021). Estas deficiencias en el proceso de enseñanza a distancia, sobre todo en comunidades rurales (Martínez y Gamboa, 2020; CEPAL, 2020; Demera et al., 2021), donde no se cuenta con las herramientas básicas para garantizar el aprendizaje y satisfacción de los estudiantes, ha generado que las medidas establecidas por las autoridades federales no hayan sido implementadas adecuadamente (Oñate y Cañas, 2021; Hamdan et al., 2021).

Aunque son pocos los estudios que se han realizado a nivel Latinoamérica, los resultados que han obtenido dan una perspectiva de las dificultades que los estudiantes han enfrentado en esta nueva modalidad de aprendizaje. Ferro et al., (2021) a través de un estudio en estudiantes de universidades chilenas, identificó resistencia por parte de estudiantes con relación a la clase de educación física en formato virtual. Otros estudios han reportado dificultades como: problemas relacionados a estrés por sobrecarga de tareas, mala implementación de cursos en línea, falta de orientación en sus universidades y poca flexibilidad por parte de los profesores (Rosario et al., 2020).

Lo anterior, contrasta con las opiniones de otros países como: Arabia Saudita, Vietnam y Nepal. Por ejemplo, Dinh y Nguyen (2020) en un estudio realizado con estudiantes de trabajo social en Vietnam, identificaron que, a pesar de haber enfrentado algunas dificultades técnicas, los estudiantes se sintieron satisfechos porque contaron con el apoyo disponible para el aprendizaje en línea. Otro estudio realizado por Almusharraf, y Khahro (2020) en estudiantes de nivel postsecundario en el Reino de Arabia Saudita, reveló que los estudiantes están satisfechos con el personal universitario y los miembros de la facultad que acordaron el uso de plataformas específicas para esta nueva modalidad de aprendizaje. Sharma et al., (2020) desarrollaron una investigación en estudiantes de Chitwan Medical College, Bharatpur en Nepal, donde detectó que el 53.5\% de los estudiantes están satisfechos con la educación en línea.

Bajo este nuevo reto educativo, el presente trabajo tiene como objetivo determinar y comparar el nivel de satisfacción y las dificultades a las que se enfrentaron los estudiantes del nivel superior del Subsistema de Universidades Tecnológicas ubicadas en zonas con diferente grado de urbanización. 
Retos y nivel de satisfacción de...

\section{METODOLOGÍA}

Este estudio se realizó bajo un enfoque cuantitativo, carácter no experimental, descriptivo comparativo, tipo transversal. El muestreo fue probabilístico aleatorio simple. Dado que las encuestas fueron aplicadas antes de que iniciara el ciclo escolar de las instituciones que se rigen por este subsistema, los únicos criterios de inclusión fueron que los estudiantes no fueran de nuevo ingreso y que estuvieran tomando clases bajo modalidad virtual. Para ello se aplicó el instrumento a estudiantes de una institución del SUTyP ubicada en una comunidad rural (UTIM) y dos ubicadas en áreas urbanas (UTP y UTJ). La selección de las dos últimas instituciones se realizó en función de la disponibilidad de las autoridades para aplicar el instrumento.

Instrumento para la recolección de datos: se elaboró un instrumento conformado por 32 ítems los cuales se agruparon de la siguiente manera: 9 sobre datos demográficos, 9 relacionados con el acceso a internet, 12 de opinión sobre el sistema de enseñanza virtual y dos sobre las principales problemáticas que han enfrentado. 25 de las preguntas fueron diseñadas para respuestas cerradas, 5 con escala de Likert y dos abiertas. El instrumento fue validado a través de una evaluación por pares previo a su aplicación.

Dado que las dos últimas preguntas del instrumento fueron abiertas, en la penúltima se les solicitó a los encuestados describir en no más de tres renglones su experiencia respecto a las limitaciones, retos y dificultades a los que se ha enfrentado durante esta pandemia. Las respuestas fueron agrupadas con base en el reto o problemática. Problemáticas descritas como: "Ha sido un reto muy difícil estar en clases en línea por las condiciones de la señal e Internet", "En mi comunidad no se cuenta con buena recepción de Internet (muy inestable) y el uso de datos de igual forma lo que provoca que en algunas sesiones no se tomen a su totalidad" o "Ha sido un reto, ya que hay horarios en los que no es posible acceder a la red, ya sea por la inclemencia del clima o falta de acceso a él en la comunidad" se agruparon como problemas de acceso.

Respuestas como las siguientes: "Ha sido una buena experiencia, sí he aprendido, pero no lo suficiente como en una clase presencial, normalmente las dudas no se resuelven completamente", "No poder resolver las dudas que surgen en clase de la forma que debería ser con el docente" o "Ha sido complicado el aprendizaje porque estoy acostumbrado a clases presenciales para entender mejor" se agruparon como problemas de aprendizaje. 
Debido a que, algunas de las respuestas más comunes fueron: "Pues un tanto difíciles ya que no se encuentra trabajo por la pandemia y dificultades para cubrir gastos tanto en casa como de la universidad", "Se me ha complicado un poco el estudiar y trabajar, por motivos de tiempo y falta de recursos por la pandemia" o "Me ha afectado económicamente ya que me ha costado pagar los cuatrimestres" se agruparon como problemas económicos.

Por otra parte, para el análisis de las respuestas a la última pregunta, estas se agruparon en 24 rubros que se encuentran en la tabla 7 del material complementario. Respuestas como: "No aprendo como debería, debido a que no siempre hay excelente señal por las dos partes o más", "En mi comunidad no se cuenta con buena recepción de Internet (muy inestable) y el uso de datos de igual forma lo que provoca que en algunas secciones no se tomen a su totalidad" o "Una de las limitaciones es trabajar con software educativos y en ocasiones existen fallas en la descarga o instalación y al estar a distancia en ocasiones los profesores no pueden solucionar ese tipo de problemas, existen fallas en el internet o en las plataformas" se agruparon dentro en el rubro de brecha tecnológica.

Finalmente, respuestas como: "Pues me vi afectado en pandemia por la muerte de mi padre, entones pues estudio y trabajo para sustentar los gastos de la casa y los míos", "Ha sido la mayor tortura de la vida, más respeto al fallecimiento de mi padre por COVID" o "Pues ha sido triste por la pérdida de familiares" se agruparon en el rubro de pérdidas humanas. Otro tipo de respuestas comunes fueron: "Es complicado poder resolver dudas y al mismo tiempo aprender, no estamos acostumbrados o tenemos la facilidad de ser autodidactas", "Uno de los mayores retos que he enfrentado, ha sido tratar de ser un poco más autodidacta y aún sigo en proceso de serlo" o "El poder aprender yo sola más cosas de mi interés ya que no estoy acostumbrada a ser autodidacta" las cuales fueron agrupadas en el rubro de falta de habilidades autodidactas. Otro tipo de respuestas que se identificaron en las encuestas fueron las relacionadas con los problemas de aprendizaje. Observando respuestas como: "Es difícil aprender en línea, se aprende, pero muy poco, por más atención que se preste", "Pues durante el periodo de clases que he tomado desde mi casa siento que no he aprendido lo suficiente con respecto a mi carrera. Necesitamos regresar a clases presenciales" o "Creo que es complicado debido a que este sistema implementado no funciona, pues hay muchos factores externos que influyen en el correcto aprendizaje, desde estrés a otros no 
relacionados con los participantes directamente" se agruparon dentro del rubro no aprende debidamente. Finalmente, una vez agrupadas las respuestas, fueron analizados mediante gráficos de barras para identificar las frecuencias de cada grupo.

Para identificar algunos aspectos relacionados con el nivel de adaptación de los estudiantes al formato virtual de las clases se incluyeron en el instrumento las siguientes preguntas: 1.¿Qué tanto se ha familiarizado con el uso de las plataformas utilizadas?, 2.Cuando le surge una duda, su profesor atiende en el momento que usted realiza la consulta, (en horarios laborables), 3.Cuando usted realiza una consulta a sus profesores, sus dudas quedan completamente esclarecidas, 4. El formato que han utilizado sus profesores para la impartición de clases ha sido suficiente para su aprendizaje, 5. Está usted de acuerdo en que las clases se sigan desarrollando de manera virtual, lo que resta del año. Mismas que se designaron como: adaptación, atención de dudas, esclarecimiento de dudas, formato y aceptación, respectivamente.

Características y tamaño de la muestra: el instrumento se aplicó a estudiantes de tres Universidades Tecnológicas (UT). La de Izúcar de Matamoros como referencia de comunidad rural, y la de Jalisco y Puebla como referencia de zonas urbanas. Para la aplicación de las encuestas se contactó al secretario académico de la UT de Puebla (UTP), a Docentes de la UT de Jalisco (UTJ) y a los directores de carrera de la UT de Izúcar de Matamoros (UTIM). Se solicitó el apoyo para atender a la encuesta por parte de los estudiantes de cada institución. Dada la respuesta de los estudiantes, se respondieron: 111, 63 y 1105 que representan el 12, 6 y 16\% de la matricula total de la UTIM, UTJ y UTP respectivamente.

Dado que para la primera pregunta se utilizó una escala del tipo: poco, mucho, más o menos y para las preguntas 2, 3, 4 y 5 se utilizó escala de Likert, se asignaron números a las respuestas. En función de estos números se realizó un análisis de varianza para identificar diferencias significativas entre los estudiantes de las tres instituciones del subsistema de universidades tecnológicas. Los valores asignados a cada respuesta se organizaron en una tabla de Excel, la cual fue exportada a una base de datos en el programa STASTISTICA para identificar diferencias significativas entre las tres instituciones mediante análisis de varianza de una sola vía, con un nivel de confianza del $95 \%$ y un valor de alfa igual a 0.05 . 
Tratamiento de los datos: las respuestas demográficas fueron organizadas en tablas de contingencia. Los datos relacionados con los ingresos semanales de los encuestados fueron representados a través de gráficos circulares. Las respuestas referentes a las asignaturas que han presentado mayor complicación durante el modelo de enseñanza virtual se organizaron en tablas de Excel, se revisó la ortografía, se homogeneizaron las respuestas y a partir de estas se elaboraron nubes de palabras.

\section{RESULTADOS Y DISCUSIÓN}

\section{Datos demográficos}

Las edades más frecuentes de los encuestados fueron: 19, 21, y 18 a 19 años para alumnos de la UTIM. UTJ y UTP, respectivamente. Con relación al género, en la UTIM se observó homogeneidad en los encuestados (43\% masculino, 52\% femenino y el resto prefirió no especificar). En la UT de jalisco respondió un mayor porcentaje de mujeres (65\%) y un mayor porcentaje de hombres en la UT de Puebla (81\%). Respecto al origen de los encuestados predomina la gente local y solo un pequeño porcentaje fue representado por estudiantes foráneos: 11.7, 15.9 y $13.7 \%$ de la UTIM, UTJ y UTP respectivamente.

La mayoría de los encuestados indicaron que eran solteros y solo un pequeño porcentaje de ellos respondieron que son casados o viven en unión libre: 7.2, 4.8 y 2.5\% para la UTIM, UTJ y UTP, respectivamente. Si bien un considerable porcentaje de los encuestados dependen de sus padres, en la UTIM se observa que el porcentaje de alumnos que dependen de sus padres es menor (59\%) en comparación con la UTJ (74\%) y la UTP $(77 \%)$.

En la tabla 1 se observa que un mayor porcentaje de estudiantes de la UTIM aportan a la economía de la familia. Asimismo, se observó un mayor porcentaje de dependientes económicos en los encuestados de la UTIM en comparación con las otras instituciones. Lo anterior es congruente, dado que algunos estudiantes de la UTIM son casados, viven en unión libre o apoyan simplemente al gasto familiar.

Tabla 1. Aportación y dependientes económicos

\begin{tabular}{|c|c|c|c|c|}
\hline \multirow{2}{*}{} & \multicolumn{2}{|c|}{ Aportación económica } & \multicolumn{2}{c|}{ Dependientes económicos } \\
\cline { 2 - 5 } & $\mathbf{S i}$ & $\mathbf{N o}$ & $\mathbf{S i}$ & No \\
\hline UTIM & $\mathbf{6 0 . 4}$ & 39.6 & $\mathbf{3 1 . 7}$ & 68.3 \\
\hline UTJ & 36.9 & 63.1 & 15.9 & 84.1 \\
\hline UTP & 42.8 & 57.2 & 20.8 & 79.2 \\
\hline
\end{tabular}


En la figura 1 se observan los gráficos correspondientes al ingreso semanal. Se muestra que entre los estudiantes de la UTIM predominan ingresos semanales familiares de $\$ 500$ a $\$ 1000$, un $76 \%$ de ellos percibe ingresos menores o iguales a $\$ 2,000$. El 64\% de los encuestados de la UTJ perciben ingresos de $\$ 500$ a $\$ 3,000$, frente al $55 \%$ de los estudiantes de la UTP que perciben de $\$ 1,100$ a $\$ 4,000$.

El 93\% de los encuestados de la UTP viven en comunidades urbanas, dicho porcentaje fue mayor en comparación con los estudiantes de la UTJ (79.2\%). Lo anterior se debe a que tanto la UTJ como la UTP están ubicadas en zonas urbanas. Dadas las características de la región de impacto de la UTIM el 49\% de los estudiantes vive en áreas urbanas y el $46 \%$ en comunidades rurales.

Figura 1. Ingresos semanales familiares de los encuestados por institución educativa

\section{UTIM}
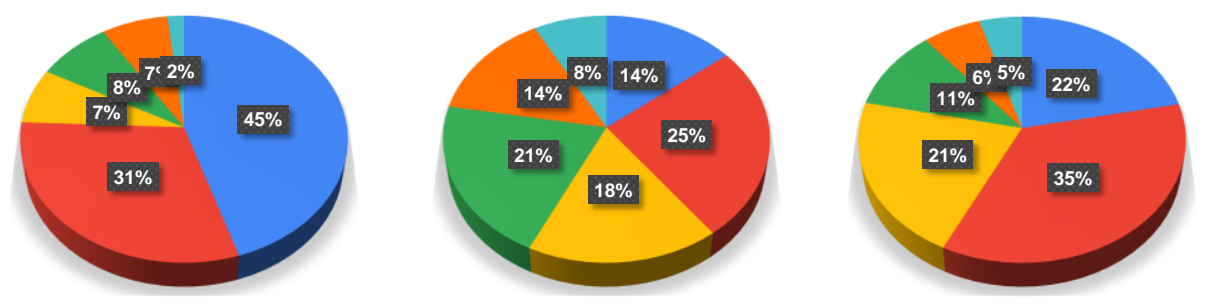

$\square 500$ a $\$ 1000$

- $\$ 1100$ a $\$ 2000$

$\$ 2100$ a $\$ 3000$

- $\$ 3100$ a $\$ 4000$

- $\$ 4100$ a $\$ 5000$

Más de $\$ 5000$

\section{Conectividad}

En la tabla 2 se observa que el porcentaje de estudiantes con cobertura de internet en sus comunidades es menor en la UTIM, en comparación con las otras dos instituciones. Esto se debe a que casi la mitad de ellos provienen de comunidades rurales. Para el caso de los estudiantes de la UTP y la UTJ, cuentan con mayor cobertura y solo un pequeño porcentaje de ellos no. Del mismo modo, la cantidad de estudiantes que cuentan con conexión de internet propia es menor en la UTIM, seguido de la UTJ y la UTP.

También se observan los datos relacionados con la conexión a internet, dispositivo disponible para tomar sus clases y el gasto en uso de datos móviles. Uno de los dispositivos más comunes para tomar clase es el celular. La disponibilidad de una computadora (propia o rentada) es de: $22.5,39.7$ y 32.8 para la UTIM, UTJ y UTP respectivamente. Debido a que la mayoría se conecta a sus clases con el celular, el 68, 63 y $62 \%$ de los estudiantes de la UTIM, UTJ y UTP respectivamente, respondieron que gastan semanalmente de \$100 a \$200 por uso de datos móviles. 
Tabla 2. Disponibilidad, tipo de dispositivo y gasto para conectarse a clases virtuales

\begin{tabular}{|l|c|c|c|}
\hline \multicolumn{1}{|c|}{ Cobertura de internet } \\
\hline $\mathrm{Si}$ & UTIM (\%) & UTJ (\%) & UTP $(\%)$ \\
\hline No & 67.6 & 93.7 & 87.9 \\
\hline Intermitente & 6.3 & 1.6 & 2.9 \\
\hline Conexión propia & 26.1 & 4.8 & 9.2 \\
\hline $\mathrm{Si}$ & 66.7 & 96.8 & 87.4 \\
\hline No & 33.3 & 15.9 & 12.6 \\
\hline Dispositivo de acceso & 73.0 & 60.3 & 65.7 \\
\hline Celular & 22.5 & 39.7 & 32.8 \\
\hline PC personal & 4.5 & 0.0 & 1.5 \\
\hline Café internet & 20.7 & 15.9 & 22.1 \\
\hline Gasto semanal en saldo para uso de datos & 44.4 & 41.7 \\
\hline \$50.00 & 47.7 & 19.0 & 20.1 \\
\hline \$100.00 & 19.8 & 20.6 & 16.1 \\
\hline \$200 & 11.7 &
\end{tabular}

Dado que no todos los estudiantes tienen posibilidades para adquirir un equipo de cómputo. El 48\%, 46 y 63 \% de los encuestados de la UTIM, UTJ y UTP respectivamente, respondieron que rentan computadoras para conectarse a sus clases. La disponibilidad de lugares con renta de PC no es la misma para todos los estudiantes, ya que cuando se les preguntó sobre el número de sitios que rentan equipos de cómputo en sus comunidades, un gran porcentaje de los encuestados (75: UTIM, 84: UTJ, 78: UTP) respondieron que no existen más tres sitios que ofrecen este servicio, cuyo costo promedio de la renta de equipo en su mayoría es de 10 a 15 pesos.

\section{Carga académica}

Los estudiantes que respondieron a la encuesta se encuentran estudiando en los siguientes programas educativos. UTIM: administración, tecnologías de la información, contaduría y gestión de negocios. UTJ: administración y mercadotecnia. UTP: sistemas automotrices, ingeniería industrial, tecnologías de la información, gastronomía, ingeniería ambiental, mecatrónica, energías renovables, administración, ingeniería automotriz, contaduría y mantenimiento industrial. 
La mayoría de los encuestados respondió que toman 8 materias. Mismas que se toman en línea de acuerdo con los horarios establecidos por su institución. La plataforma más utilizada es Google meet, y en la figura 2 se observa que las asignaturas que más se les complican son aquellas que tienen que ver con habilidades matemáticas, inglés y aquellas materias en las que se requiere hacer prácticas como las asignaturas de especialidad e integradora.

Figura 2. Nubes de palabras con las asignaturas con mayor grado de dificultad en formato virtual, de acuerdo con la frecuencia de las respuestas.
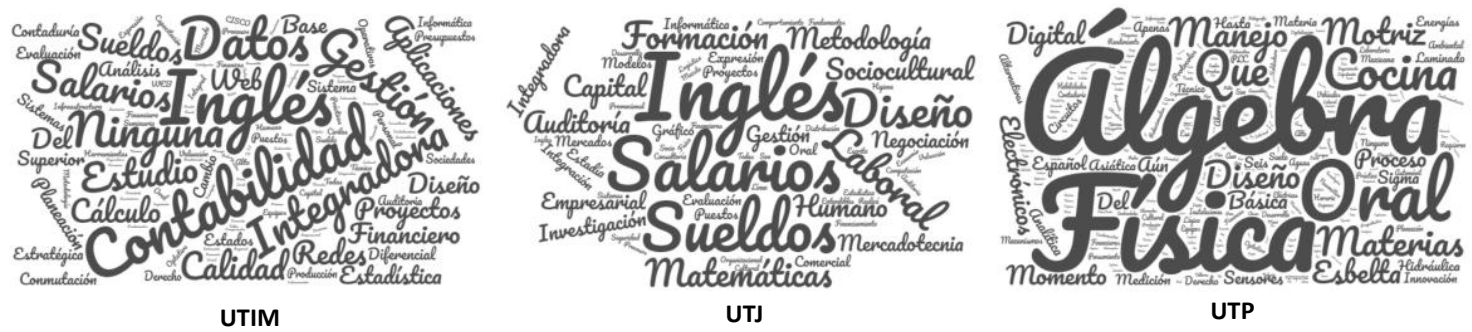

\section{Retos y dificultades de la comunidad estudiantil}

En la figura 3 se observan tres principales problemáticas que son: conexión a internet (acceso), aprendizaje y problemas económicos. Con relación a los problemas de acceso, los encuestados respondieron que tuvieron problemas para conectarse a sus clases debido a: la falta de conexión propia, fallas en el servicio de internet, no contar con un dispositivo para atender las clases adecuadamente, o gastos excesivos para la compra de datos móviles.

Respecto a la segunda causa, refieren que no se aprende lo mismo en este formato, toda vez que ellos estaban acostumbrados a las clases presenciales. De la misma manera, mencionaron que cuando tienen dudas no siempre es posible resolverlas debido a la poca disposición de sus docentes. Finalmente, algo que enfatizaron fue que las asignaturas de especialidad o aquellas que requieren de prácticas no fueron comprendidas en el formato virtual.

Algunos estudiantes respondieron que ven a este nuevo modelo de enseñanza virtual como un reto y que ellos saben que deben adaptarse a las circunstancias. Incluso algunos de ellos comentaron que han desarrollado habilidades que no tenían al comienzo de la pandemia. En contraparte, algunos de los alumnos mencionaron que han padecido estrés o ansiedad debido a que por el confinamiento no pueden ver a sus amigos o practicar algún deporte. 
Figura 3. Retos y dificultades de los estudiantes ante el modelo de enseñanza virtual

\section{UTIM}
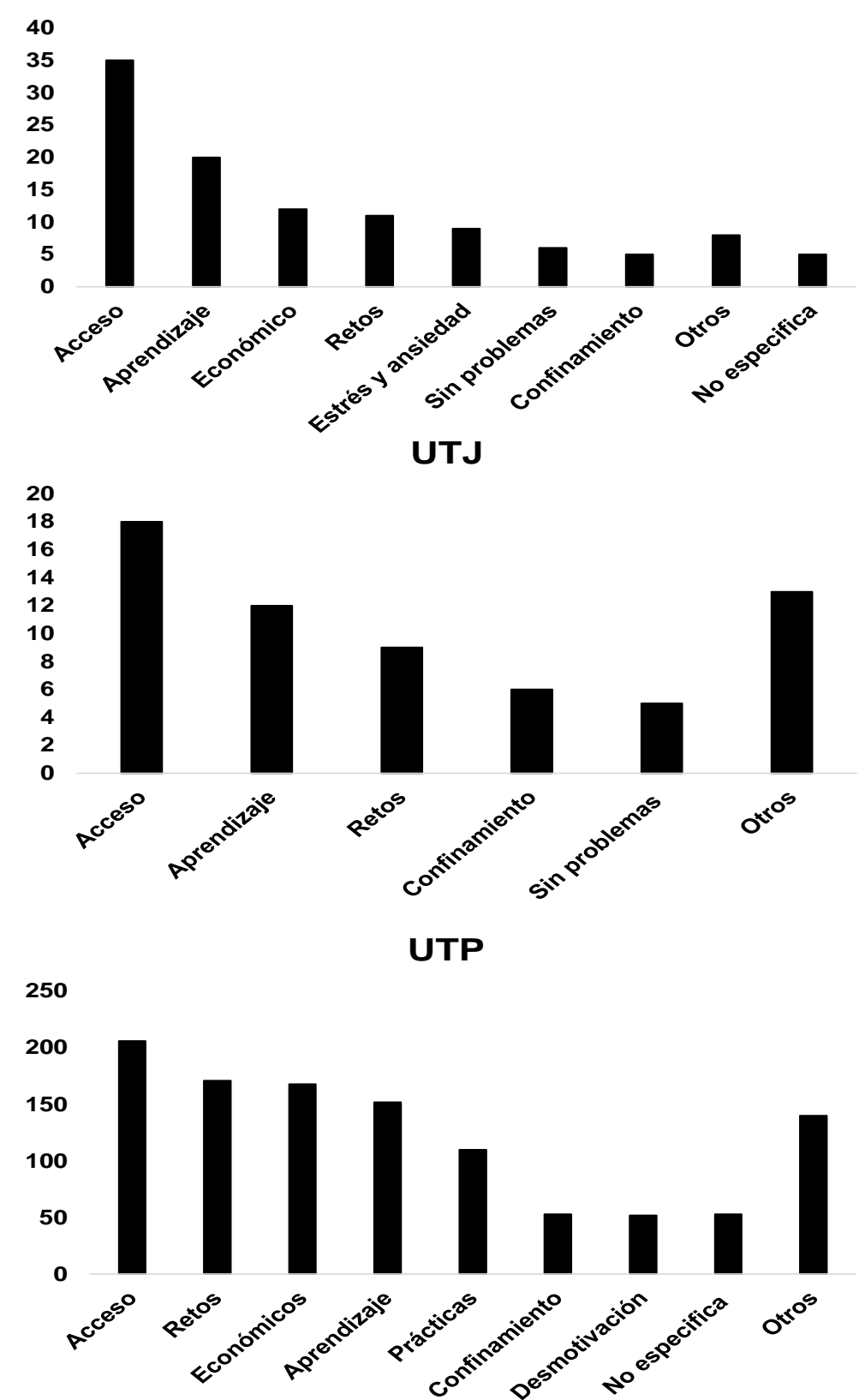

\section{Experiencias y opiniones ante el modelo de enseñanza virtual}

En la figura 3 se observan los principales retos y experiencias de los encuestados de la UTIM, UTJ y UTP. Uno de los retos que tuvieron en común las tres instituciones es la falta de conexión a internet, mala calidad del servicio, falta de un dispositivo para conectarse o falta de cobertura en sus comunidades. Entre las experiencias más frecuentes entre los estudiantes de la UTP y la UTIM fueron los problemas económicos. Lo cual surgió a raíz de la muerte del jefe de familia, pérdida de empleo, disminución de las ventas 
(sobre todo en aquellos que tienen algún negocio) o a que algunos de ellos son casados y tienen que solventar los gastos de la familia.

Los resultados observados en este trabajo coinciden con los reportados por otros autores.

Ordorika (2020), menciona que este nuevo modelo de enseñanza representa grandes retos tecnológicos, pedagógicos y de competencias. Asimismo, representa una "oportunidad importante para proponer posibilidades de aprendizajes más flexibles, explorar aprendizajes híbridos o mezclados y combinar aprendizajes sincrónicos y asincrónicos. Por otra parte, Calvo et al., (2020), señalaron que este nuevo modelo de enseñanza ha impactado en la perspectiva que los estudiantes tienen sobre los docentes, mientras que estos señalan la falta de habilidades tecnológicas por parte de los estudiantes de nivel superior. Gómez y Escobar (2021), hacen referencia a que esta nueva modalidad ha dejado en evidencia las desigualdades educativas. Concluyendo que la educación en tiempos de pandemia se ha convertido en un privilegio al que solo tienen acceso aquellos que cuentan con las condiciones sociales, tecnológicas y económicas, en desmedro de los grupos vulnerables; con lo que se incrementan las brechas de desigualdad social.

FIGURA 4. Retos y experiencias durante el modelo de educación virtual

UTIM

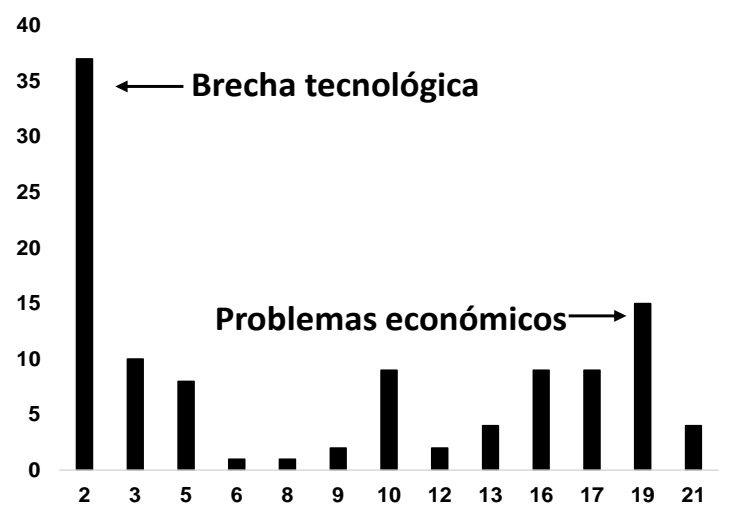

UTJ

20

UTP

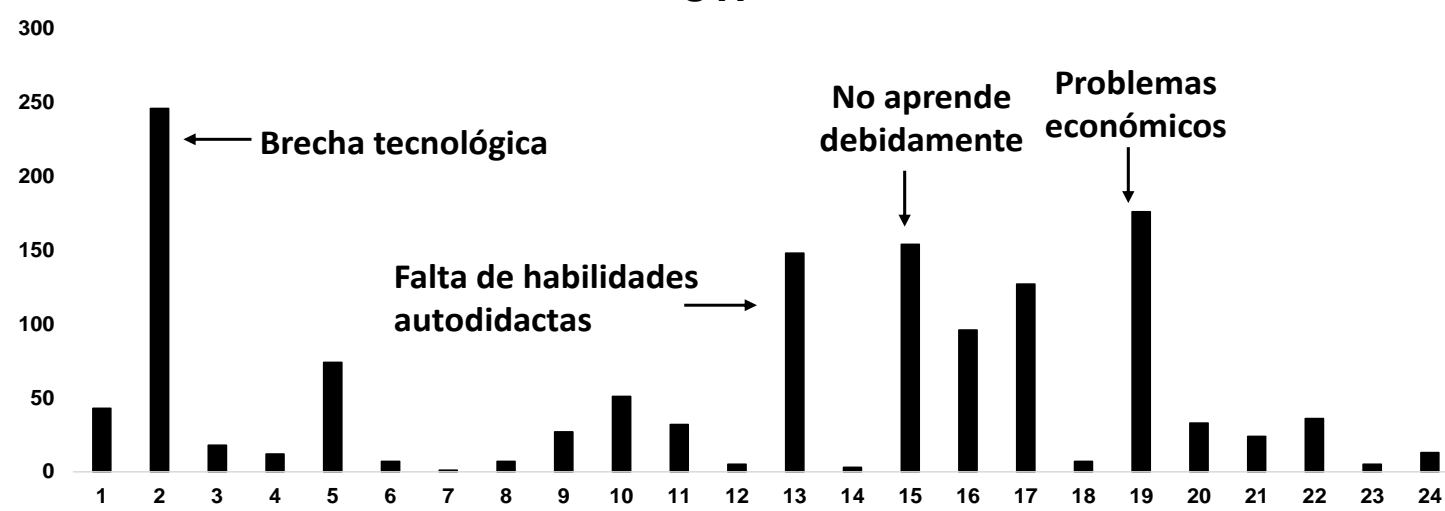


La brecha tecnológica como una de las principales problemáticas durante este nuevo modelo de enseñanza han sido un común denominador en diversos estudios publicados. Domínguez y López (2021) reportan que, si bien en las comunidades rurales se tiene acceso a la telefonía celular, no todos los docentes y alumnos poseen las mismas condiciones de acceso, disposición, digitalización y alfabetización tecnológica. Rojas y Quezada (2021), también reportaron el avance desigual de la digitalización como uno de los principales problemas. Erreyes y Álvarez (2021) mencionan que no todos los estudiantes tienen acceso al internet y no se conectan a las clases virtuales; en este contexto es necesario que los docentes mejoren las estrategias de enseñanza para obtener mejores niveles de satisfacción en los estudiantes.

También respondieron que el aprendizaje no es el mismo, sobre todo en aquellas materias de especialidad o las que precisan de prácticas. Algunos comentaron que en el lugar donde toman sus clases existen muchos distractores que no les permiten concentrase en el tema que se está impartiendo. Otras personas manifestaron la poca disposición de los docentes para atender las dudas, así como casos en los que el docente solo lee las diapositivas o no se ve su interés por garantizar que los alumnos comprendan el tema que se está desarrollando (7, 3, 4.5 y 3.1\%de los encuestados de la UTIM, UTJ y UTP, respectivamente).

El $13.4 \%$ de los estudiantes de la UTP indicaron que falta desarrollar habilidades autodidactas. Sobre todo, para estar preparados para este tipo de circunstancias donde no queda más que adaptarse al modelo de educación virtual, como una estrategia de prevención de contagios en la comunidad estudiantil. Por otra parte, el 8, 8.1 y $11 \%$ de los encuestados de la UTIM, UTJ y UTP, respectivamente han encontrado el lado positivo de esta pandemia, sobre todo para desarrollar habilidades en el manejo de plataformas virtuales y algunas herramientas básicas para la elaboración, edición y entrega de tareas.

\section{Nivel de adaptación al modelo de enseñanza virtual y apoyo docente}

En la figura 5 se observan los gráficos correspondientes al análisis de varianza que se aplicó a las respuestas sobre el nivel de adaptación y satisfacción ante el modelo de enseñanza virtual. Se observa que en ninguno de los dos aspectos se observaron diferencias estadísticamente significativas con un nivel de confianza del $95 \%$ y un valor de $\alpha=0.05$ los estudiantes de las tres instituciones dieron respuestas entre más o menos, o mucho respecto a la adaptación al modelo virtual. 
A pesar de que los resultados obtenidos con relación al nivel de satisfacción no mostraron diferencias estadísticamente significativas. Se observa que los estudiantes de la UTP están más satisfechos con el modelo virtual, esto coincide con las respuestas dadas en preguntas anteriores, donde comentaron que, si bien estar trabajando bajo un modelo virtual no es tan cómodo y eficiente como el presencial, están conscientes que debido a la pandemia y como acción preventiva es mejor atender las clases de manera virtual.

Figura 5. Nivel de adaptación y satisfacción ante el modelo de enseñanza virtual

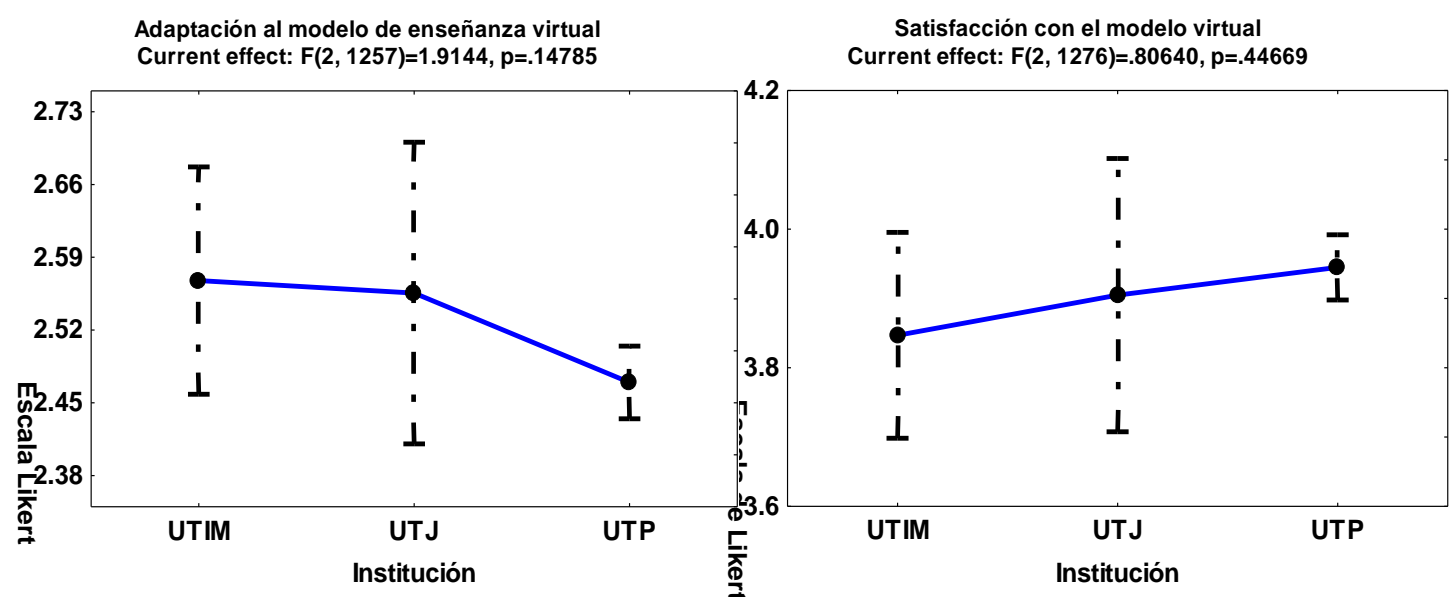

Ante este modelo de enseñanza virtual, es necesario monitorear la atención de los docentes para con los estudiantes. Es por lo que se les preguntó sobre el apoyo de sus docentes ante alguna duda y la manera en que sus cuestionamientos son aclarados. Ambos aspectos mostraron diferencias estadísticamente significativas. Obteniendo mayor satisfacción de los docentes de la UTP, UTJ y UTIM. A pesar en las preguntas anteriores algunos estudiantes de la UTP comentaron que no estaban de acuerdo con la forma de enseñanza de sus profesores, un porcentaje mayor de ellos manifestó que sus docentes han mostrado disponibilidad para aclarar las dudas que surgen de la clase.

Figura 6. Nivel de satisfacción ante el apoyo docente y aclaración de dudas

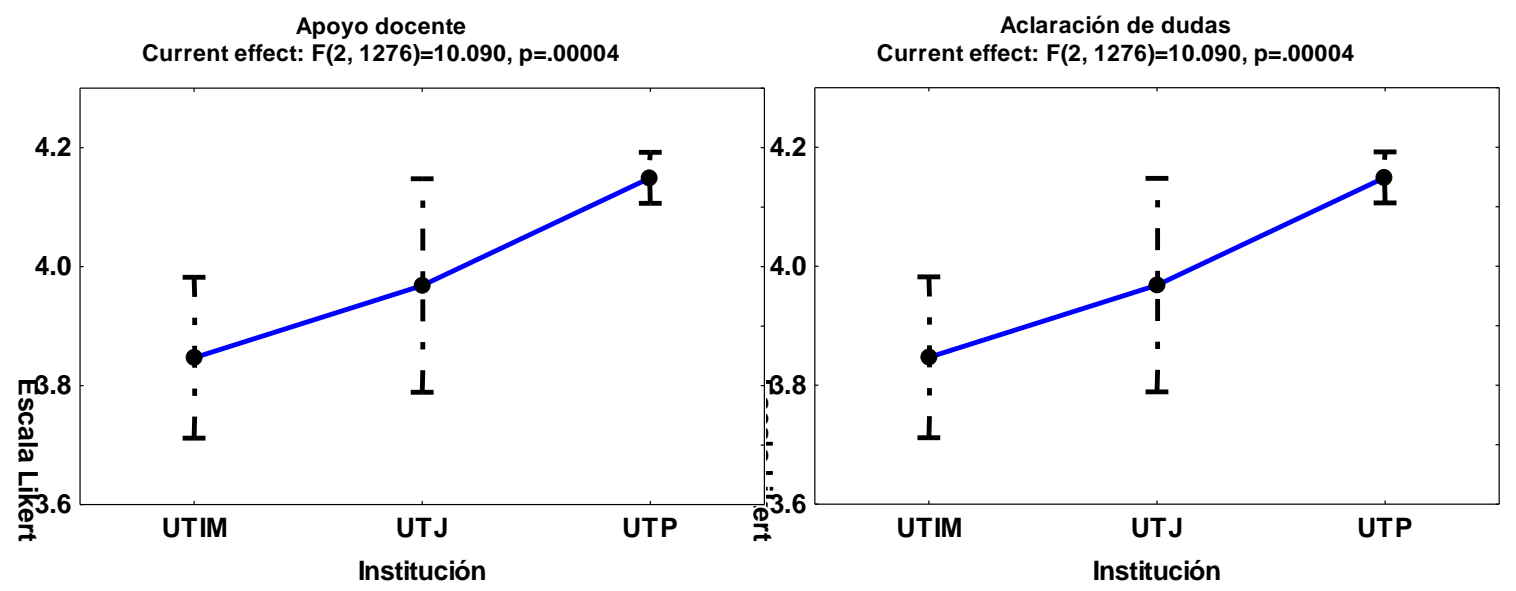


También se les preguntó si estaban de acuerdo en continuar con este modelo de enseñanza virtual. Aunque no se observaron diferencias estadísticamente significativas entre las tres instituciones. Los estudiantes de la UTJ fueron quienes respondieron en mayor porcentaje, que están de acuerdo en continuar con este modelo (Figura 7 del material complementario).

\section{CONCLUSIONES}

Los retos y dificultades que los estudiantes de las comunidades rurales han enfrentado durante este modelo de enseñanza virtual están relacionados principalmente con los siguientes aspectos: la escasa cobertura tecnológica y la falta de un dispositivo adecuado para conectarse a las clases. El ingreso semanal de los estudiantes en las comunidades rurales es inferior al de los que estudian en regiones con un mayor grado de urbanización. Si bien los estudiantes de instituciones ubicadas en zonas más urbanizadas perciben mayores ingresos. La pérdida de empleo, muerte del jefe de familia o número de dependientes económicos, y falta de un dispositivo adecuado para conectarse, han ocasionado un aprendizaje deficiente.

Debido a la falta de empleo y los bajos salarios que prevalecen en las comunidades rurales, los estudiantes deben trabajar para poder aportar a la economía del hogar, lo que los imposibilita para poder atender debidamente las clases virtuales, menguando sus capacidades de aprendizaje.

Derivado de los problemas de acceso y atención a clases virtuales. Los estudiantes no están satisfechos debido a que no aprenden debidamente, sobre todo en las asignaturas que requieren del desarrollo de prácticas. Si bien los estudiantes también mencionaron que las asignaturas que más se les complicaron fueron aquellas que están relacionadas con matemáticas, este no es un problema que haya surgido con el actual modelo de enseñanza, sino que ha sido una dificultad recurrente en estudiantes de todos los niveles educativos.

\section{REFERENCIAS BIBLIOGRAFICAS}

Almusharraf, N., \& Khahro, S. (2020). Students satisfaction with online learning experiences during the COVID-19 pandemic. International Journal of Emerging Technologies in Learning (IJET), 15(21), 246-267.

Calvo, S. T., Cervi, L., Tusa, F., y Parola, A. (2020). Educación en tiempos de pandemia: reflexiones de alumnos y profesores sobre la enseñanza virtual universitaria en 
España, Italia y Ecuador. Revista Latina de Comunicación Social, (78), 1-21. https://www.doi.org/10.4185/RLCS2020-1466

CEPAL, N. (2020). La educación en tiempos de la pandemia de COVID-19. Recuperado el de julio de 2021, de https://repositorio.cepal.org/bitstream/handle/11362/45904/1/S2000510_es.pdf

Demera-Zambrano, K. C., López-Vera, L. S., y Santana-Mero, R. C. (2021). Análisis del Plan Educativo Aprendamos Juntos en Casa para la continuidad educativa en zonas rurales. Dominio de las Ciencias, 7(2), 502-520.

Diario Oficial de la Federación (2020). ACUERDO número 02/03/20 por el que se suspenden las clases en las escuelas de educación preescolar, primaria, secundaria, normal y demás para la formación de maestros de educación básica del Sistema Educativo Nacional, así como aquellas de los tipos medio superior y superior dependientes de la Secretaría de Educación Pública. Recuperado el 6 de agosto de 2021 ,

https://www.dof.gob.mx/nota_detalle.php?codigo $=5589479 \&$ fecha $=16 / 03 / 2020$

Dinh, L. P., \& Nguyen, T. T. (2020). Pandemic, social distancing, and social work education: Students' satisfaction with online education in Vietnam. Social Work Education, 39(8), 1074-1083.

Dress G. F. y Zhang P. (12 de agosto de 2021). El escaso acceso digital frena a América Latina y el Caribe ¿Cómo solucionar este problema? BANCO MUNDIAL BLOGS. Recuperado el 14 de julio de 2021, de: https://blogs.worldbank.org/es/latinamerica/el-escaso-acceso-digital-frena-americalatina-y-el-caribe-como-solucionareste?cid=ECR_E_NewsletterWeekly_ES_EXT\&deliveryName=DM114206

Erreyes-Toledo, N. M., y Álvarez-Lozano, M. I. (2021). Perspectivas de los estudiantes de educación rural en tiempos de pandemia. CIENCIAMATRIA, 7(13), 46-65.

Fernández, M., Herrera, L., Hernández, D., Nolasco, R., and De la Rosa, R. (2020). Lecciones del Covid-19 para el sistema educativo mexicano. Nexos. Distancia por tiempos. Blog de educación. Recuperado el 14 de junio de 2021, de https://educacion.nexos.com.mx/lecciones-del-covid-19-para-el-sistema-educativomexicano/ 
Ferro, E. F., Cid, F. M., Briceño, M. H., Duarte, S. A. G., Salse, Á. R. S., Troncoso, S. P., y Olivos, C. L. (2021). Nivel de satisfacción de las clases online por parte de los estudiantes de Educación Física de Chile en tiempos de pandemia. Retos: nuevas tendencias en educación física, deporte y recreación, (41), 123-130.

Figueroa, T. A., Castro, J. M., Calderón, A. I., y Alburqueque, C. A. (2021). Escuelas rurales en el Perú: factores que acentúan las brechas digitales en tiempos de pandemia (COVID-19) y recomendaciones para reducirlas. Educación, 30(58). https://doi.org/10.18800/educacion.202101.001

Gómez-Arteta, I., y Escobar-Mamani, F. (2021). Educación virtual en tiempos de pandemia: incremento de la desigualdad social en el Perú. Chakiñan, Revista de Ciencias Sociales y Humanidades. S. (15), 152-165. https://doi.org/10.37135/chk.002.15.10 Guach, R. A. D., Tejeda, J. J. G., y Abreu, M. R. P. (2020). Características clínico-epidemiológicas de la COVID-19. Revista Habanera de Ciencias Médicas, 19 (2), 1-15.

Hamdan, K. M., Al-Bashaireh, A. M., Zahran, Z., Al-Daghestani, A., Samira, A. H., \& Shaheen, A. M. (2021). University students' interaction, Internet self-efficacy, selfregulation and satisfaction with online education during pandemic crises of COVID19 (SARS-CoV-2). International Journal of Educational Management. 35 (3), 713 725. https://doi.org/10.1108/IJEM-11-2020-0513

Martínez, Y., y Gamboa, L. A. A. (2020). Covid-19, pobreza y educación en Chiapas: análisis a los programas educativos emergentes. Revista Internacional de Educación para la Justicia Social, 9(3), 61-82.

Mendiola, M. S., Hernández, A., Torres, R., Carrasco, M. D. A. S., Romo, A., Mario, A., y Cazales, V. (2020). Retos educativos durante la pandemia de COVID-19: una encuesta a profesores de la UNAM. Revista Digital Universitaria, 21(3), 1-24.

Mishra, L., Gupta, T., \& Shree, A. (2020). Online teaching-learning in higher education during lockdown period of COVID-19 pandemic. International Journal of Educational Research 100012. https://doi.org/10.1016/j.ijedro.2020.100012

Oñate, M. G., y Cañas, D. (2021). Las desigualdades sistémicas del Sistema de Educación Intercultural Bilingüe se profundizan durante la crisis sanitaria del COVID-19 
Retos y nivel de satisfacción de...

(Investigaciones). Revista Andina de Investigación. 4(1), 65-72. https://repositorio.uasb.edu.ec/handle/10644/7959

Ordorika, I. (2020). Pandemia y educación superior. Revista de la educación superior, 49(194), 1-8. https://doi.org/10.36857/resu.2020.194.1120

Rojas, L. P., y Quezada, A. N. (2021). Educación a distancia y pandemia. Experiencias en educación básica en zonas rurales y urbanas. Reencuentro. Análisis de problemas universitarios, 31(78), 101-122.

Rosario-Rodríguez, A., González-Rivera, J. A., Cruz-Santos, A., y Rodríguez-Ríos, L. (2020). Demandas tecnológicas, académicas y psicológicas en estudiantes universitarios durante la pandemia por COVID-19. Revista Caribeña de Psicología, 4(2), 176-185. https://doi.org/10.37226/rcp.v4i2.4915

Ruiz Larraguivel, E. (2009). La construcción de una nueva ocupación. Los técnicos con escolaridad superior del Sistema de Universidades Tecnológicas. Diferenciación de la educación superior. Sus relaciones con el mundo laboral, México, ISSUE/Plaza y Valdés.

Sharma, K., Deo, G., Timalsina, S., Joshi, A., Shrestha, N., \& Neupane, H. C. (2020). Online learning in the face of COVID-19 pandemic: Assessment of students' satisfaction at Chitwan medical college of Nepal. Kathmandu University Medical Journal, 18(2), 40-47.

Suarez, V., Quezada, M. S., Ruiz, S. O., y De Jesús, E. R. (2020). Epidemiología de COVID-19 en México: del 27 de febrero al 30 de abril de 2020. Revista clínica española, 220(8), 463-471.

Thomas, C. J. (2020). Coronavirus and challenging times for education in developing countries. Washington DC: The Brookings Institution. Recuperado el 24 de agosto de 2021, de https://www.brookings.edu/blog/education-plusdevelopment/2020/04/13/coronavirus-and-challenging-times-for-education-indeveloping-countries/ 